\title{
Comparison of the suitability of a 200 ha land for rice cultivation by three 'native' techniques in Southern Nigeria.
}

\author{
Umweni, A. $\mathrm{S}^{1}$. and Ogunkunle, A.O ${ }^{2}$. \\ ${ }^{1}$ Department of Soil science, Edo State University, Ekpoma, Edo State, Nigeria \\ ${ }^{2}$ Department of Agronomy, University of Ibadan, Ibadan, Nigeria
}

\begin{abstract}
A 200 hectare parcel of land in Edo state of Nigeria was evaluated for its suitability for rice cultivation, under three land utilization types (LUT):(i) Rain-fed upland,(ii) Natural flood and (iii) Irrigated rice cultivation. Six soil mapping units were established and appropriate guidelines specific for each LUT was followed in establishing the suitability of the land for rice cultivation. The suitability of each soil for each of the different land utilization types was ranked relative to the other soils and the rankings were compared using the Spearman's ranking correlation coefficient. The results showed that Rain-fed upland rice can only be practiced in 40 hectares (19.5\%) of the area, at a marginal class of suitability, Irrigation can be practiced in at least 160 ha and Natural floods in 189 ha (94.5\%). With a correlation coefficient of 0.96 between Irrigated and Natural flood methods, it means that either of them can be practiced but because their seasons are different, one can be made to follow the other in practice - Irrigation to follow Natural floods.
\end{abstract}

Key words: Rice suitability, Rain-fed, Natural flood, Irrigation (LUT).

\section{Introduction.}

The Illushi people are a rice growing community and earn their living principally through rice farming work. Some of them cultivate the crop on upland with natural rain, others depend on the natural yearly floods from the Niger River while others use irrigation as a third option. Each of these Land Utilization types (LUT) has its effect on the soil and eventually on the yield of rice. There is therefore the need to assess the soils of the community in order to determine which of the land utilization type(s) the soils can best support on a sustainable basis.

The role of rice in the world's food budget cannot be overestimated. It is the staple food for nearly half of the world population as well as being a major source of employment and income for many especially the rural populace (FAO, 2003)[1]. I It is estimated that some 156 million hectares of land are in rice cultivation across the world with a combined production capacity of about 660 million tons (Genctan, 2009)[2], approximately $4.23 \mathrm{t} / \mathrm{ha}$. Out of this, Nigeria is credited with 1.77 million hectares with an insignificant contribution from Edo State, thus the State's potentials remain largely untapped (Longtau, 2003)[3]. For Nigeria to make appreciable contribution to the world's rice production, the land utilization type must be that for which the soils of the rice producing regions are well suitable for. This is the essence of land evaluation.

Many guidelines have been provided by many authors on the soil/land conditions for the cultivation of rice such as those by Tyagi (2003)[4] for paddy rice, FAO (1993)[5] for bunded rice, Prakash (2003)[6] and Odengiz et al (2010)[7] for upland rice and Sys (1985)[8] for upland, natural flood and irrigated rice. The method to be adopted in this study is that according to the guidelines provided by Sys (1985)[8] as we seek to evaluate the suitability of the soils of the Illushi community for the land utilization types most commonly practiced by the people. It affords the opportunity to compare the relevance of the land utilizations in use and recommend the most relevant for adoption.

\subsection{Location.}

\section{Materials and methods.}

This is a 200 hectare site located at the "Ega" end of what is referred to as Illushi mainland, in Esan north east local government area of Edo State. It lies between latitudes $06^{0} 41^{1} \mathrm{~N}$ to $06^{0} 41^{1} 58^{\prime \prime} \mathrm{N}$ and longitudes $06^{0} 35^{1} \mathrm{E}$ to $06^{0} 36^{1} \mathrm{E}$. Rainfall from the nearest meteorological station shows that rainfall is unstable, low and the commencement of rainy season is often very unpredictable. While in some years, rains may commence as early as April, in some others it is as late as July. However, it is reported that rainfall is about $1200 \mathrm{~mm} /$ year. Flooding pattern is equally as unpredictable as the rainfall. It is seasonally flooded from the River Niger resulting in the alluvial deposits from which the soils are largely derived. The distribution pattern of rainfall is such that the area can be without rain for as long as 5-6 months (November - April). Relative Humidity varies from about $70 \%$ in the rainy season to about $55-60 \%$ in the dry season. Temperature is generally high. It can be as high as $34{ }^{\circ} \mathrm{C}$ in the dry season but as low as $15{ }^{\circ} \mathrm{C}$ at nights in the harmattan period. Vegetation is guinea savannah characterised by numerous grass species and scattered shrubs. Andropogon spp. and Pennisetum are 
common grasses. Fairly big trees occur along the river/stream courses. Because of the prolonged dry season, bush burning has become an established practice for reducing the drudgery of manual land preparation by the natives. Generally, the land is flat with very few microtopographical differences and the soils are derived from the alluvial deposits of the River Niger.

\subsection{Field work and Laboratory studies.}

Soil identification and mapping was by the rigid grid method with the aid of the soil auger. A predetermined format of $50 \mathrm{~m} \times 50 \mathrm{~m}$ was adopted thus: transverses were cut at $50 \mathrm{~m}$ apart along a predetermined baseline and observation points were at $50 \mathrm{~m}$ apart along the traverses. Auger holes were made at each point down to a depth of $120 \mathrm{~cm}$ and soil was examined at each of $0-15 \mathrm{~cm}, 15-30 \mathrm{~cm}, 30-60 \mathrm{~cm}, 60-90 \mathrm{~cm}$ and $90-120 \mathrm{~cm}$ depths. Morphological properties were recorded at each point, including texture, drainage, colour, slope, evidence of erosion, rock outcrop etc. The points were then grouped on the basis of the recorded morphological features. A total of 6 profile pits $(150-200 \mathrm{~cm}$ deep) were dug at points typical of each mapping unit. The profiles were described following FAO guidelines (FAO, 1977b)[9]. Samples were collected from all the horizons of each profile for laboratory analysis.

\subsection{Laboratory analysis.}

This involved routine and general methods as summarized in Table 1:

Table 1: Laboratory analytical methods employed for soil analysis.

\begin{tabular}{|c|c|}
\hline Soil variable & Method \\
\hline $\mathrm{pH}\left(\mathrm{H}_{2} \mathrm{O}\right)$ & $1: 2$, soil:water ratio \\
\hline Available P & $\begin{array}{l}\text { Bray I (Bray and Kurtz, 1945)[10], the P in solution was measured by the } \\
\text { molybdenum blue method on the Technicon autoanalyzer. }\end{array}$ \\
\hline Organic C (OC) & Wet oxidation method (Walkley and Black, 1934)[11] \\
\hline Total N & Berthelot reaction using a Technicon autoanalyzer (Technicon AAll, 1971)[12]. \\
\hline Exchangeable acidity (EA) & $1 \mathrm{MKCl}$ extraction \\
\hline $\begin{array}{l}\text { Effective Cation-exchange } \\
\text { capacity (ECEC) }\end{array}$ & Summation of exchangeable cations and exchangeable acidity (Tan, 1996)[13]. \\
\hline $\begin{array}{l}\text { Exchangeable cations }(\mathrm{Ca}, \mathrm{Mg} \text {, } \\
\mathrm{K}, \mathrm{Na})\end{array}$ & $\begin{array}{l}\text { Extraction using } \mathrm{NH}_{4} \mathrm{OAC} \text { buffered at } \mathrm{pH} 7.0 \text { (Thomas, 1982)[14].Ca and } \mathrm{Mg} \text { (AAS) } \\
\text { and } \mathrm{K} \& \mathrm{Na} \text { (Flame photometer) }\end{array}$ \\
\hline $\begin{array}{l}\text { Particle-size distribution (sand, } \\
\text { silt, clay) }\end{array}$ & Hydrometer method. \\
\hline Base saturation & Calculated as the sum of exchangeable bases divided by ECEC multiplied by 100 . \\
\hline
\end{tabular}

\section{Land suitability evaluation procedure.}

The properties of each soil mapping unit was matched with the requirement of the land land utilization type(i.e. rain-fed upland rice, irrigated rice, rice by natural floods ) to assign a suitability class to it following the guidelines provided by Sys (1985)[8],( tables 2, 3 and 4, ). The eventual suitability class of a mapping unit for the LUT is the class indicated by the characteristic with the lowest rating, in line with the "Law of Minimum" approach (FAO, 1984)[15].

The suitability classes of each of the soils for the land utilization types were ranked and the rankings were compared using the Spearman's ranking correlation coefficient. 
Table 2: Land/soil requirements for rain -fed upland rice

\begin{tabular}{|c|c|c|c|c|c|}
\hline \multirow[t]{2}{*}{ Land characteristics } & \multicolumn{3}{|c|}{ Land } & \multirow{2}{*}{ 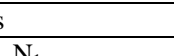 } & \multirow[b]{2}{*}{$\mathrm{N}_{2}$} \\
\hline & $\mathrm{S}_{1}$ & $\mathrm{~S}_{2}$ & $\mathrm{~S}_{3}$ & & \\
\hline $\begin{array}{l}\text { Climate (C ): } \\
\text {--Rainfall (mm) }\end{array}$ & $>1,400$ & $>1,000$ & $>800$ & $<800$ & $<800$ \\
\hline $\begin{array}{l}\text { Topography(t): } \\
\text {--Slope }(\%) \quad(1) \\
(2)\end{array}$ & $\begin{array}{l}<4 \\
<8\end{array}$ & $\begin{array}{l}<8 \\
<16\end{array}$ & $\begin{array}{l}<16 \\
<30\end{array}$ & $\begin{array}{l}<25 \\
<30\end{array}$ & $\begin{array}{l}>25 \\
>30\end{array}$ \\
\hline $\begin{array}{l}\text { Wetness (w): } \\
\text {--Flooding } \\
\text {--Drainage (3) } \\
\text { (4) }\end{array}$ & $\begin{array}{l}\text { Nil } \\
\text { Good } \\
\text { Imperfect }\end{array}$ & $\begin{array}{l}\text { Nil } \\
\text { Moderate or better } \\
\text { Imperfect } \\
\text { moderate }\end{array}$ & $\begin{array}{l}\text { Nil to slight } \\
\text { Imperfect or better } \\
\text { Good, imperfect or } \\
\text { moderate }\end{array}$ & $\begin{array}{l}\text { Nil to slight } \\
\text { Poor or better } \\
\text { Poor or better }\end{array}$ & $\begin{array}{l}\text { Any } \\
\text { Very poor } \\
\text { or better } \\
\text { Very poor } \\
\text { or better }\end{array}$ \\
\hline
\end{tabular}

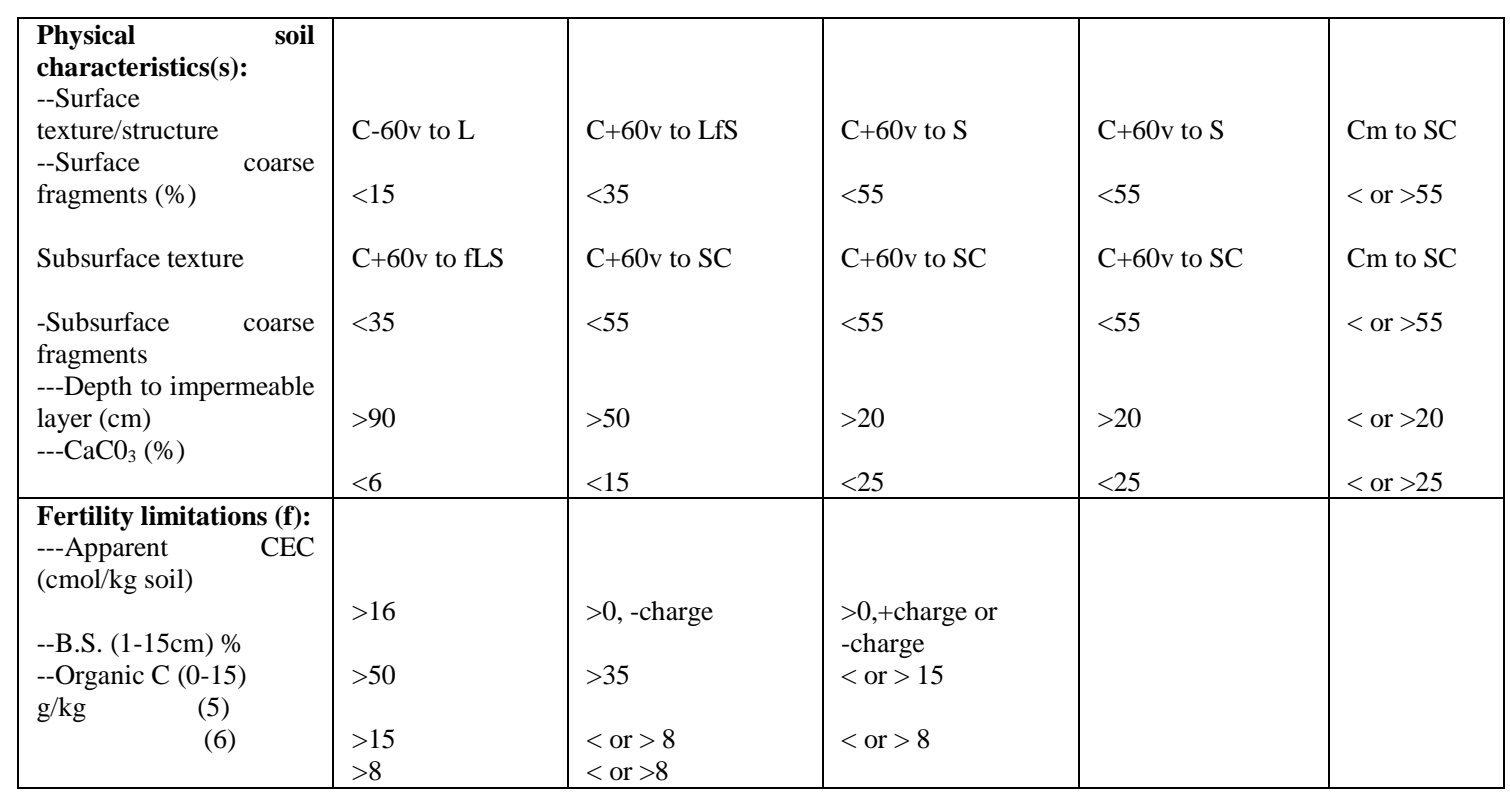

(1)= Intensive fully mechanized agriculture; (2) = Primitive farming; (3) Fine, loamy or clayey families; (4) = Coarse, loamy and sandy families; $(5)=$ Non-calcareous soils; $(6)=$ Calcareous soils.

SOURCE: Sys (1985)[8].

Table 3: Land/soil requirements for irrigated rice

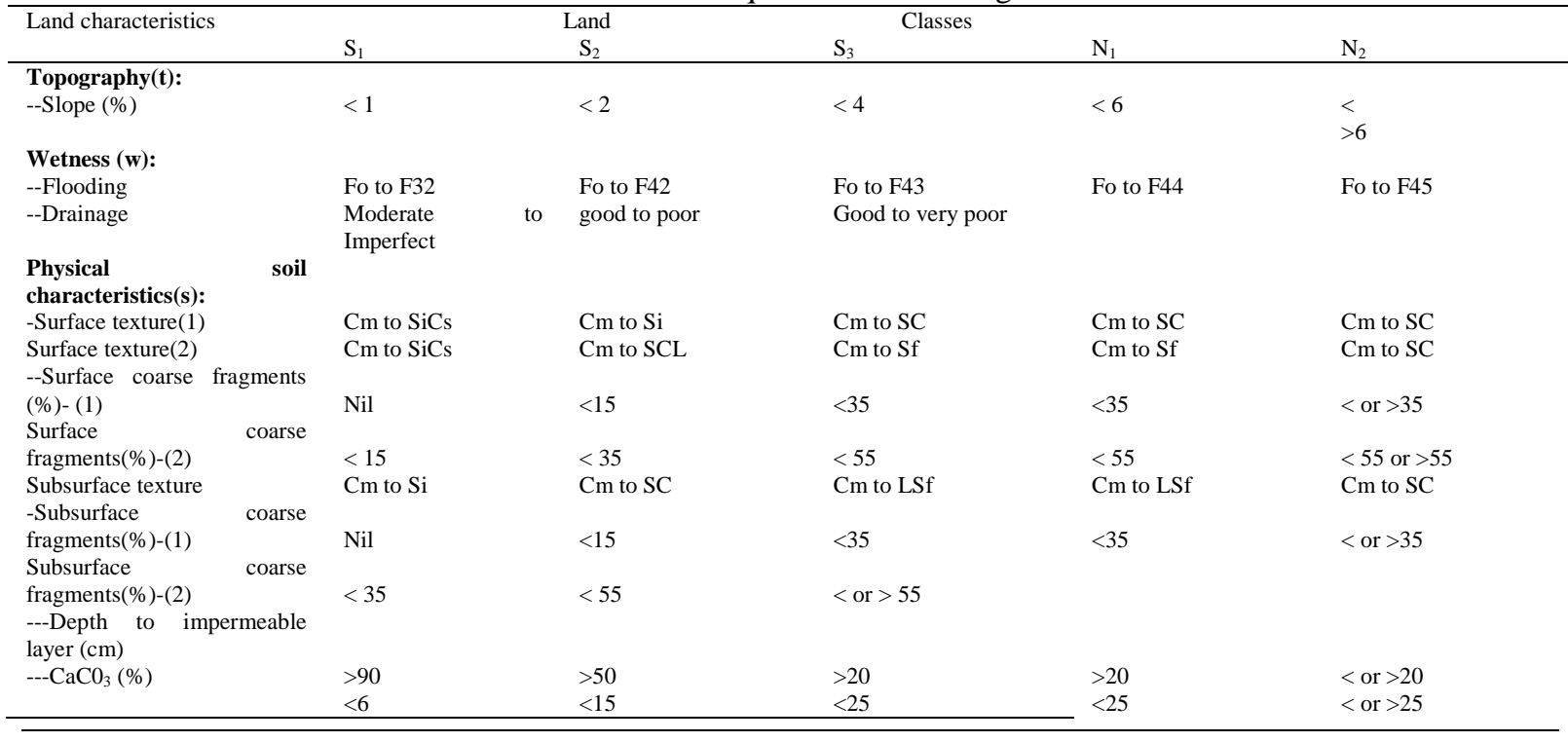


Fertility limitations (f)

---Apparent CEC $(\mathrm{cmol} / \mathrm{kg}$

soil)

$\begin{array}{rlll}\text {--B.S. }(1-15 \mathrm{~cm}) \% & >16 & >0,- \text { charge } & >0,+ \text { charge or } \\ \text {--Organic C (0-15) } & & & \text {-charge } \\ \text { g/kg } \begin{aligned} \text { (5) } \\ \text { (6) }\end{aligned} & >50 & >35 & <\text { or }>15 \\ & >15 & <\text { or }>8 & <\text { or }>8\end{array}$

$\begin{array}{lll}>15 & <\text { or }>8 & <\text { or }>8 \\ >8 & <\text { or }>8 & \end{array}$

(1) = Intensive fully mechanized agriculture; (2) = Primitive farming; (3) Fine, loamy or clayey families; (4) $=$ Coarse, loamy and sandy families; $(5)=$ Non-calcareous soils; $(6)=$ Calcareous soils .

SOURCE: Sys (1985)[8].

III. Results and Discussion.

The suitability of the various soils, as represented by the six pedons, for the three land utilization types LUT) for rice cultivation is discussed based on the guidelines in tables 2, 3 and 4 , the laboratory results in table 5 and the evaluation tables for the three LUTs in tables 6,7 and 8.

Table 5 - Physical and chemical properties of the pedons at Illushi/Ega.

\begin{tabular}{|c|c|c|c|c|c|c|c|c|c|}
\hline \multirow[b]{2}{*}{ Pedon } & \multirow[b]{2}{*}{ Horizon? } & \multirow[b]{2}{*}{ Depth $(\mathrm{cm})$} & \multirow{2}{*}{$\begin{array}{l}\text { Sand } \\
\leftarrow \\
\leftarrow\end{array}$} & \multirow{2}{*}{$\begin{array}{l}\text { Silt } \\
\mathrm{g} / \mathrm{kg} \\
\end{array}$} & \multirow{2}{*}{$\begin{array}{l}\text { Clay } \\
\rightarrow \\
\end{array}$} & \multirow[b]{2}{*}{$\mathrm{pH}\left(\mathrm{H}_{2} \mathrm{O}\right)$} & \multirow{2}{*}{$\begin{array}{l}\mathrm{Na} \\
\leftarrow \\
\end{array}$} & \multirow{2}{*}{$\begin{array}{l}\mathrm{K} \\
\mathrm{cmol} / \mathrm{kg}\end{array}$} & \multirow{2}{*}{$\begin{array}{l}\mathrm{Ca} \\
\rightarrow \\
\end{array}$} \\
\hline & & & & & & & & & \\
\hline \multirow[t]{5}{*}{$20 / 1$} & & $0-11$ & 490 & 144 & 366 & 5.5 & 0.09 & 0.14 & 10.96 \\
\hline & & $11-36$ & 470 & 84 & 446 & 5 & 0.07 & 0.12 & 6 \\
\hline & & $36-68$ & 710 & 44 & 246 & 5 & 0.07 & 0.06 & 4.64 \\
\hline & & $68-133$ & 560 & 94 & 346 & 5 & 0.09 & 0.09 & 2.56 \\
\hline & & $133-180$ & 630 & 94 & 276 & 4.8 & 0.09 & 0.07 & 2.4 \\
\hline \multicolumn{10}{|l|}{$3 / 3$} \\
\hline & & $0-14$ & 580 & 144 & 276 & 4.8 & 0.12 & 0.36 & 6.56 \\
\hline & & $14-40$ & 520 & 94 & 386 & 4.6 & 0.1 & 0.17 & 5.92 \\
\hline & & $40-100$ & 720 & 54 & 226 & 4.8 & 0.07 & 0.09 & 4.16 \\
\hline & & $100-122$ & 760 & 64 & 176 & 4.5 & 0.06 & 0.07 & 4 \\
\hline & & $122-180$ & 750 & 54 & 196 & 4.9 & 0.07 & 0.07 & 2.16 \\
\hline \multicolumn{10}{|l|}{$4 / 2$} \\
\hline & & $0-10$ & 560 & 224 & 216 & 4.8 & 0.13 & 0.23 & 11.04 \\
\hline & & Oct-40 & 430 & 184 & 386 & 5 & 0.12 & 0.18 & 6.48 \\
\hline & & $40-95$ & 680 & 54 & 266 & 5 & 0.07 & 0.07 & 4 \\
\hline & & $95-160$ & 800 & 74 & 126 & 4.8 & 0.07 & 0.07 & 2.8 \\
\hline & & $160-210$ & 680 & 44 & 276 & 4.5 & 0.07 & 0.07 & 2.4 \\
\hline \multicolumn{10}{|l|}{$19 / 11$} \\
\hline & & $0-14$ & 880 & 54 & 66 & 4.8 & 0.18 & 0.1 & 2.64 \\
\hline & & $14-38$ & 860 & 34 & 106 & 4.8 & 0.16 & 0.09 & 2.4 \\
\hline & & $38-81$ & 960 & 14 & 26 & 5 & 0.15 & 0.13 & 2.4 \\
\hline & & $81-142$ & 870 & 24 & 106 & 4.9 & 0.14 & 0.2 & 3.68 \\
\hline & & $142-180$ & 800 & 14 & 186 & 5 & 0.16 & 0.21 & 2.72 \\
\hline \multicolumn{10}{|l|}{$2 / 9$} \\
\hline & & $0-12$ & 740 & 37 & 223 & 5 & 0.02 & 0.05 & 1.92 \\
\hline & & $12-35$ & 750 & 27 & 223 & 5 & 0.03 & 0.09 & 1.6 \\
\hline & & $35-87$ & 900 & 27 & 73 & 5.2 & 0.04 & 0.07 & 2.24 \\
\hline & & 87155 & 920 & 17 & 63 & 5.4 & 0.03 & 0.07 & 3.2 \\
\hline & & $155-185$ & 790 & 17 & 193 & 5.2 & 0.04 & 0.07 & 2.32 \\
\hline \multirow[t]{6}{*}{$5 / 8$} & & $0-18$ & 680 & 97 & 223 & 5 & 0.03 & 0.09 & 2 \\
\hline & & $18-37$ & 730 & 67 & 203 & 5.1 & 0.03 & 0.05 & 1.84 \\
\hline & & $37-73$ & 890 & 57 & 53 & 5.4 & 0.02 & 0.03 & 2.16 \\
\hline & & $73-108$ & 880 & 37 & 83 & 5.4 & 0.02 & 0.03 & 3.92 \\
\hline & & $108-133$ & 840 & 37 & 123 & 5.4 & 0.02 & 0.02 & 0.72 \\
\hline & & $133-170$ & 750 & 37 & 213 & 5.3 & 0.02 & 0.04 & 2.56 \\
\hline
\end{tabular}


Title: Comparison of the suitability of a 200 ha land for rice cultivation by three 'native' techniques

\begin{tabular}{|c|c|c|c|c|c|c|c|c|c|}
\hline $\mathrm{Mg}$ & $A l$ & EA & ECEC & & $\mathrm{N}$ & $\mathrm{C}$ & & & Textural \\
\hline$\leftarrow$ & & $\mathrm{cmol} / \mathrm{kg}$ & $\rightarrow$ & $\mathrm{P}(\mathrm{mg} / \mathrm{kg})$ & $\leftarrow \mathrm{g} / \mathrm{kg}$ & $\rightarrow$ & $\mathrm{dS} / \mathrm{m}$ & & (TC) \\
\hline 1.68 & 4 & 4.4 & 17.27 & 2.94 & 0.08 & 2.56 & 3.9 & & SC \\
\hline 2.8 & 4.5 & 6.4 & 15.39 & 2.21 & 0.11 & 1.09 & 0.89 & & $\mathrm{SC}$ \\
\hline 0.56 & 3.6 & 4.4 & 9.73 & 2.21 & 0.06 & 0.38 & 1.9 & & SCL \\
\hline 0.8 & 4.8 & 6.3 & 9.84 & 4.41 & 0.03 & 0.22 & 0.99 & & SCL \\
\hline 1.28 & 5.4 & 6.6 & 10.44 & 2.21 & 0.03 & 0.19 & 7.6 & & SCL \\
\hline 1.84 & 1.5 & 2.7 & 11.58 & 3.68 & 0.11 & 1.98 & 7.3 & & SCL \\
\hline 3.2 & 3 & 4 & 13.39 & 2.21 & 0.11 & 1.06 & 4.2 & & $\mathrm{SC}$ \\
\hline 3.12 & 7.2 & 10.3 & 17.74 & 1.47 & 0.05 & 0.32 & 0.78 & & SCL \\
\hline 1.84 & 3.9 & 4.9 & 10.87 & 2.21 & 0.05 & 0.26 & 0.82 & & SL \\
\hline 1.76 & 2.5 & 3.2 & 7.26 & & 0.05 & 0.13 & 2.8 & & SL \\
\hline 0.96 & 1.7 & 1.9 & 14.26 & 16.91 & 0.03 & 3.04 & 2.94 & & SCL \\
\hline 3.68 & 5.9 & 7.4 & 17.86 & 2.94 & 0.08 & 0.86 & 2.7 & & SC \\
\hline 1.44 & 4.4 & 7.4 & 12.98 & 3.68 & 0.11 & 0.38 & 1.7 & & SCL \\
\hline 1.68 & 2 & 2.9 & 7.52 & 4.41 & 0.14 & $0.23 / 0.13$ & 0.89 & & LS \\
\hline 1.76 & 3.5 & 5.2 & 9.5 & 1.47 & 0.1 & 0.22 & 1.3 & & SCL \\
\hline 0.24 & 1.3 & 1.6 & 4.76 & 5.88 & 0.1 & 0.9 & 4.7 & & S \\
\hline 0.32 & 1.4 & 1.7 & 4.67 & 7.35 & 0.11 & 0.48 & 0.88 & & LS \\
\hline 0.4 & 0.4 & 0.5 & 3.58 & 2.94 & 0.1 & 0.13 & 0.82 & & $S$ \\
\hline 1.76 & 1.7 & 1.8 & 7.58 & 2.21 & 0.08 & 0.22 & 0.82 & & LS \\
\hline 2.4 & 3 & 3.6 & 9.09 & 2.21 & 0.08 & 0.29 & 0.88 & & SL \\
\hline 0.16 & 0.18 & 0.2 & 2.35 & 3.33 & 0.15 & 1.34 & 5.18 & & SCL \\
\hline 0.32 & 2.2 & 2.6 & 4.64 & 2.7 & 0.05 & 0.61 & 27.17 & & SCL \\
\hline 0.16 & 0.7 & 0.9 & 3.41 & 3.3 & 0.01 & 0.51 & 8.75 & & $\mathrm{~S}$ \\
\hline 0.32 & 0.95 & 1.2 & 4.82 & 0 & 0.01 & 0.19 & 5.78 & & $S$ \\
\hline 0.16 & 3.1 & 3.8 & 6.39 & 2 & 0.01 & 0.13 & 8.57 & & SL \\
\hline 0.24 & 2.8 & 3.2 & 5.56 & 2.38 & 0.13 & 1.57 & 26 & & SCL \\
\hline 0.4 & 3.5 & 4.1 & 6.42 & 0 & 0.04 & 0.61 & 6.97 & & SCL \\
\hline 0.24 & 4.2 & 5.2 & 7.65 & 6.7 & 0 & 0.03 & 6.52 & & $S$ \\
\hline 0.16 & 3.52 & 4.4 & 8.53 & 7.32 & 0.01 & 0.1 & 28.12 & & $S$ \\
\hline 0.24 & 0.62 & 0.8 & 1.8 & 2.7 & 0.01 & 0.16 & 8.4 & & LS \\
\hline 0.64 & 1.84 & 2.3 & 5.56 & 1.33 & 0.03 & 0.13 & 251.43 & & SCL \\
\hline
\end{tabular}

$\mathrm{S}=$ Sand $; \mathrm{LS}=$ Loamy sand; $\mathrm{SL}=$ Sandy loam; $\mathrm{SCL}=$ Sandy clay loam; $\mathrm{SC}=$ Sandy clay $; \mathrm{ESP}=$ Exchangeable sodium percentage.

Table 6: Suitability classification for rain -fed upland rice

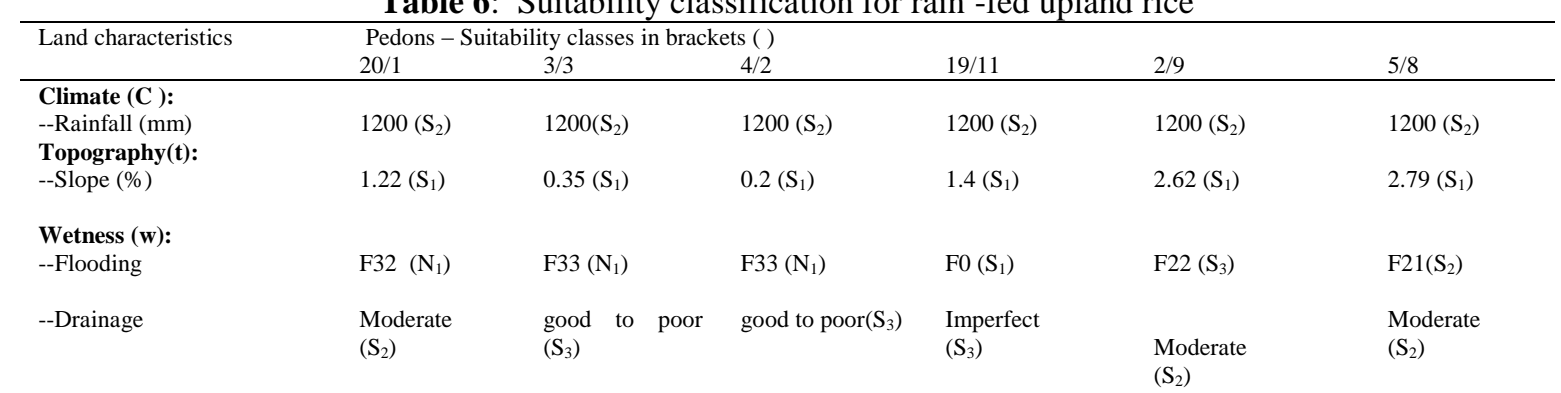


Physical soil

characteristics(s):

--Surface texture/structure

Subsurface texture

\begin{tabular}{|c|c|c|c|c|}
\hline $\mathrm{SC}\left(\mathrm{S}_{1}\right)$ & $\mathrm{SCL} / \mathrm{SC}\left(\mathrm{S}_{1}\right)$ & $\mathrm{SCL} / \mathrm{SC}\left(\mathrm{S}_{1}\right)$ & $\mathrm{S} / \mathrm{LS}\left(\mathrm{S}_{3}\right)$ & $\operatorname{SCL}\left(\mathrm{S}_{1}\right)$ \\
\hline
\end{tabular}

-Subsurface coarse

$\operatorname{SCL}\left(\mathrm{S}_{1}\right) \quad \mathrm{SL}\left(\mathrm{S}_{1}\right)$

$\operatorname{LS} / \mathrm{SCL}\left(\mathrm{S}_{2}\right)$

$\mathrm{LS} / \mathrm{SL}\left(\mathrm{S}_{2}\right)$

$\operatorname{SCL}\left(\mathrm{S}_{1}\right)$

$\mathrm{LS} / \mathrm{SCL}\left(\mathrm{S}_{2}\right)$

fragments

$\operatorname{Nil}\left(\mathrm{S}_{1}\right) \quad \mathrm{Nil}\left(\mathrm{S}_{1}\right)$

$\operatorname{Nil}\left(\mathrm{S}_{1}\right)$

$\operatorname{Nil}\left(\mathrm{S}_{1}\right)$

$\mathrm{S} / \mathrm{SL}\left(\mathrm{S}_{2}\right)$

or $>55$

---Depth
layer $(\mathrm{cm})$

$>180\left(\mathrm{~S}_{1}\right) \quad>180\left(\mathrm{~S}_{1}\right)$

$>180\left(\mathrm{~S}_{1}\right)$

$>180\left(\mathrm{~S}_{1}\right)$

$\operatorname{Nil}\left(S_{1}\right)$

$\operatorname{Nil}\left(\mathrm{S}_{1}\right)$

$\operatorname{Nil}\left(\mathrm{S}_{1}\right) \quad \operatorname{Nil}\left(\mathrm{S}_{1}\right) \quad \operatorname{Nil}\left(\mathrm{S}_{1}\right) \quad$ Nil $\left(\mathrm{S}_{1}\right)$

(1)

$>180\left(\mathrm{~S}_{1}\right)$

$\operatorname{Nil}\left(\mathrm{S}_{1}\right)$

$>180\left(\mathrm{~S}_{1}\right)$

$\operatorname{Nil}\left(S_{1}\right)$

Fertility limitations (f):

---Apparent CEC $(\mathrm{cmol} / \mathrm{kg}$

soil)

$--B . S .(1-15 \mathrm{~cm}) \%$
--Organic C $(0-15)$

$\begin{array}{lll}10-17\left(\mathrm{~S}_{1}\right) & 7-17\left(\mathrm{~S}_{1}\right) & 8-18\left(\mathrm{~S}_{1}\right) \\ 74.5\left(\mathrm{~S}_{1}\right) & 76.7\left(\mathrm{~S}_{1}\right) & 86.7\left(\mathrm{~S}_{1}\right) \\ 2.6\left(\mathrm{~S}_{2}\right) & 2.0\left(\mathrm{~S}_{2}\right) & 3.0\left(\mathrm{~S}_{2}\right)\end{array}$

$4-9\left(S_{3}\right)$

$2-6\left(S_{3}\right)$

$2-9\left(\mathrm{~S}_{3}\right)$

$\mathrm{g} / \mathrm{kg} \quad(5)$

$2.6\left(\mathrm{~S}_{2}\right)$

$2.0\left(\mathrm{~S}_{2}\right)$

$3.0\left(\mathrm{~S}_{2}\right)$

66.4( $\left.\mathrm{S}_{1}\right)$

42.4( $\left(\mathrm{S}_{2}\right)$

$1.6\left(\mathrm{~S}_{3}\right)$

$91.5\left(\mathrm{~S}_{1}\right)$

$1.0\left(\mathrm{~S}_{3}\right)$

$1.3\left(\mathrm{~S}_{3}\right)$

Aggregate class

Area (ha)

$\mathrm{N}_{1}(\mathrm{w})$

105
52.5

$\mathrm{N}_{1}(\mathrm{w})$

30

$\%$

15

25
12.5

$\mathrm{S}_{3}$ (fw)

$\mathrm{S}_{3}$ (fsw)

11

$\begin{array}{ll}11 & 23 \\ 5.5 & 11.5\end{array}$

(1) = Intensive fully mechanized agriculture; (2) = Primitive farming; (3) Fine, loamy or clayey families; (4) $=$ Coarse, loamy and sandy families; $(5)=$ Non-calcareous soils; $(6)=$ Calcareous soils.

Table 7: suitability classification for irrigated rice

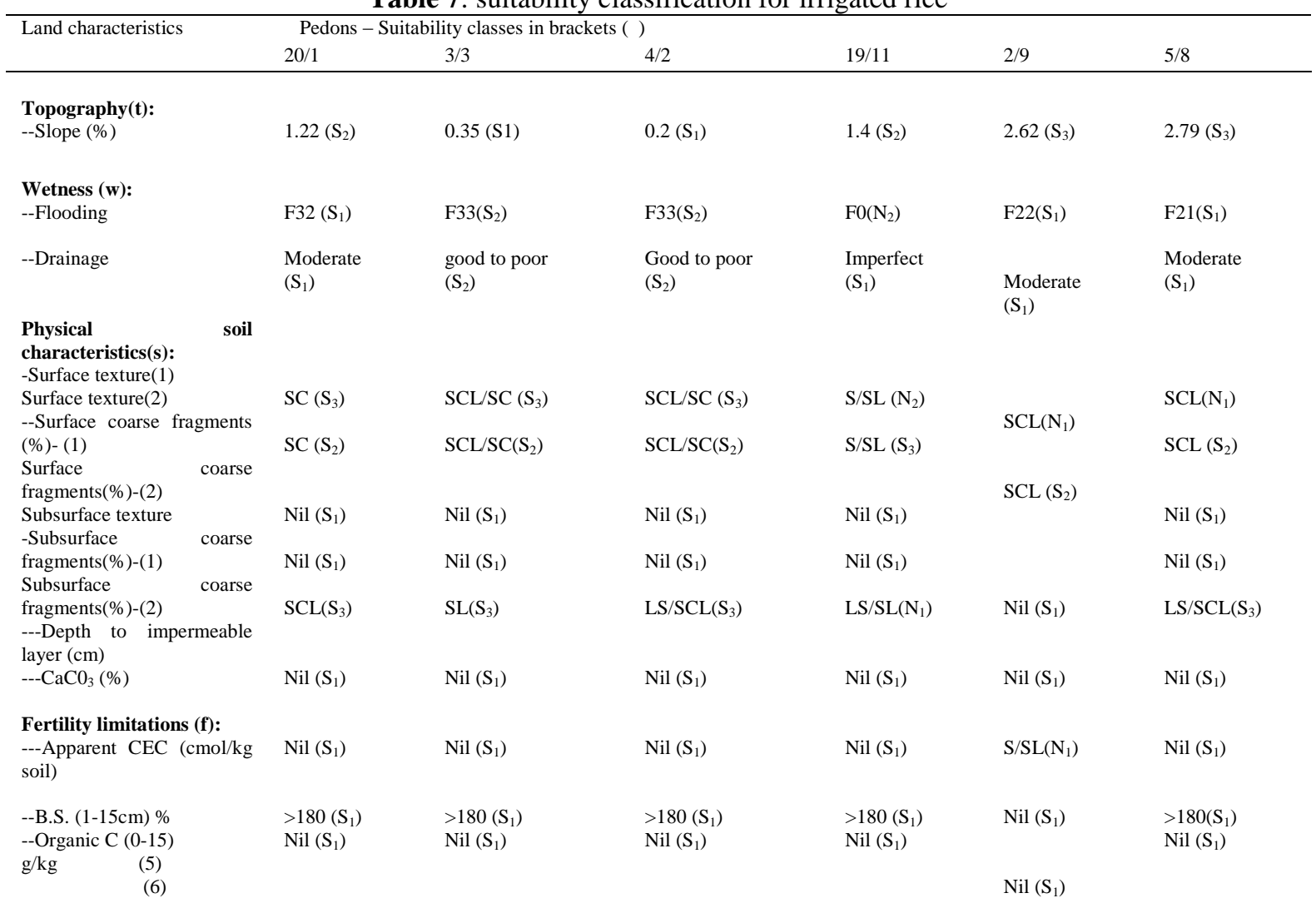




\begin{tabular}{|c|c|c|c|c|c|c|}
\hline & $10-17\left(\mathrm{~S}_{1}\right)$ & 7-17 $\left(S_{2}\right)$ & $8-18\left(S_{1}\right)$ & $4-9\left(S_{3}\right)$ & \multirow{2}{*}{$\begin{array}{l}>180\left(\mathrm{~S}_{1}\right) \\
\operatorname{Nil}\left(\mathrm{S}_{1}\right)\end{array}$} & $2-9\left(\mathrm{~S}_{3}\right)$ \\
\hline & $74.5\left(\mathrm{~S}_{1}\right)$ & $76.7\left(\mathrm{~S}_{1}\right)$ & $86.7\left(\mathrm{~S}_{1}\right)$ & $66.4\left(\mathrm{~S}_{1}\right)$ & & $42.4\left(\mathrm{~S}_{1}\right)$ \\
\hline & $2.6\left(\mathrm{~S}_{3}\right)$ & $2.0\left(\mathrm{~S}_{3}\right)$ & $3.0\left(\mathrm{~S}_{3}\right)$ & $1.0\left(\mathrm{~S}_{3}\right)$ & & $1.6\left(\mathrm{~S}_{3}\right)$ \\
\hline & & & & & $2-6\left(S_{3}\right)$ & \\
\hline & & & & & $91.5\left(\mathrm{~S}_{1}\right)$ & \\
\hline & & & & & $1.3\left(\mathrm{~S}_{3}\right)$ & \\
\hline Aggregate class & $\mathrm{S}_{3}(\mathrm{fs})$ & $\mathrm{S}_{3}(\mathrm{fs})$ & $\mathrm{S}_{3}(\mathrm{fs})$ & $\mathrm{N}_{2}(\mathrm{fsw})$ & $\mathrm{N}_{1}(\mathrm{fst})$ & $\mathrm{N}_{1}$ (fst) \\
\hline Area (ha) & 105 & 30 & 25 & 11 & 23 & 6 \\
\hline Percentage (\%) & 52.5 & 15 & 12.5 & 5.5 & 11.5 & 3 \\
\hline
\end{tabular}

(1) = Intensive fully mechanized agriculture; (2) = Primitive farming; (3) Fine, loamy or clayey families; (4) = Coarse, loamy and sandy families; (5) = Non-calcareous soils; (6) = Calcareous soils.

Table 8: Suitability classification for rice cultivation under natural floods.

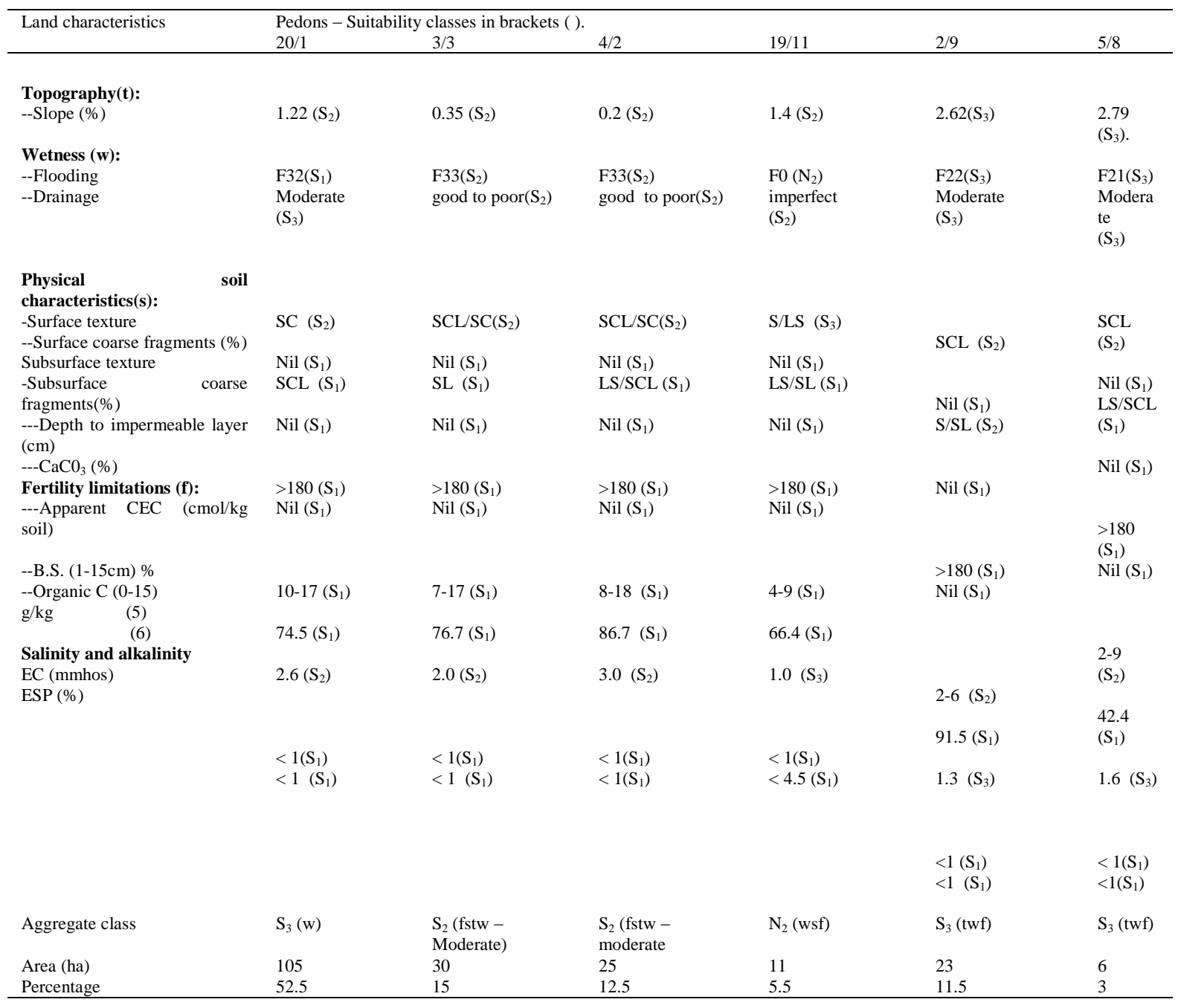

1= Intensive fully mechanized agriculture; (2) = Primitive farming; (3) Fine, loamy or clayey families; (4) = Coarse, loamy and sandy families; (5) = Noncalcareous soils; $(6)=$ Calcareous soils. SOURCE: Sys (1985)[8].

Flood sequence: F32 - F31 - F33 - F41 - F42 - F34 - F22 - F23 - F43 - F24 - F44 - F35 - F25 - F45 - F11 - F12 - F13 - F14 - F15 - Fo. 
Table 9 : Suitability groupings of the soils for the three LUTS for rice

\begin{tabular}{|c|c|c|c|c|c|}
\hline Pedons & \multicolumn{3}{|c|}{ Land suitability classes for the cultivation methods } & Area (ha) & $\%$ \\
\hline $3 / 3$ & $\mathrm{~N}_{1}(\mathrm{w})$ & $\mathrm{S}_{3}(\mathrm{fs})$ & $S_{2}$ (fstw-moderate) & 30 & 15 \\
\hline $4 / 2$ & $\mathrm{~N}_{1}(\mathrm{w})$ & $\mathrm{S}_{3}(\mathrm{fs})$ & $\mathrm{S}_{2}$ (fstw-moderate) & 25 & 12.5 \\
\hline 2/9 & $\mathrm{S}_{3}(\mathrm{fw})$ & $\mathrm{N}_{1}$ (fst) & $\mathrm{S}_{3}(\mathrm{ftw})$ & 23 & 11.5 \\
\hline $5 / 8$ & $S_{3}(f)$ & $\mathrm{N}_{1}$ (fst) & $\mathrm{S}_{3}(\mathrm{ftw})$ & 6 & 3 \\
\hline
\end{tabular}

Table 10 :Ranking of the soils by the Land suitability groupings of the soils for the LUTS

\begin{tabular}{|c|c|c|c|c|c|}
\hline \multirow[t]{2}{*}{ Pedons } & \multicolumn{3}{|c|}{ Ranks of the soils by the LUTS. } & \multirow[t]{2}{*}{ Area (ha) } & \multirow[t]{2}{*}{$\%$} \\
\hline & Rainfed & Irrigated & Natural floods & & \\
\hline $20 / 1$ & $4(5)$ & $1(2)$ & 3 & 105 & 52.5 \\
\hline $3 / 3$ & $4(5)$ & $1(2)$ & $1(1.5)$ & 30 & 15 \\
\hline $19 / 11$ & 3 & 6 & 6 & 11 & 5.5 \\
\hline $2 / 9$ & 2 & $4(4.5)$ & $4(4.5)$ & 23 & 11.5 \\
\hline $5 / 8$ & 1 & $4(4.5)$ & $4(4.5)$ & 6 & 3 \\
\hline
\end{tabular}

Table 11: Rank correlation matrix for the suitability groupings of the LUTS.

Irrigated

Rainfed

$-0.56 \mathrm{NS}$

Irrigated

$$
\begin{aligned}
& * * * \quad=\text { Significant at } 0.5 \% \\
& \text { NS }=\text { Not significant. }
\end{aligned}
$$

\subsection{Discussion:}

2.4.1. Rain-fed upland rice.

The guideline for the evaluation of the land for suitability for the above LUT is contained in table 2 and the result is detailed in table 6 and expressed in figure 1 . The results showed that about 40 hectares or $19 \%$ of the total land area is marginally suitable for the cultivation of rain-fed upland rice with limitations bothering mainly on wetness (drainage), soils and fertility. Some 29 hectares of this (pedons 2/9 and 5/8 areas) can have their limitations easily corrected by improved fertility measures and minor drainage operations to improve their current ratings to moderate levels. The remaining 160 hectares or $81 \%$ of total land area was found to be currently not suitable due to the major limitation of wetness (poor drainage) occasioned by the susceptibility of the area (pedons 20/1,3/3 and 4/2) to sustained yearly floods from the river Niger. It is hoped that if the Federal government of Nigeria's plan to dredge the river materializes, this defect would be removed and the rating of the soils will be greatly improved. Thus at current ratings it is only about 29 hectares that can be relied upon as being really marginally suitable for upland rice cultivation from 200 hectare portion of Illushi/Ega farm.

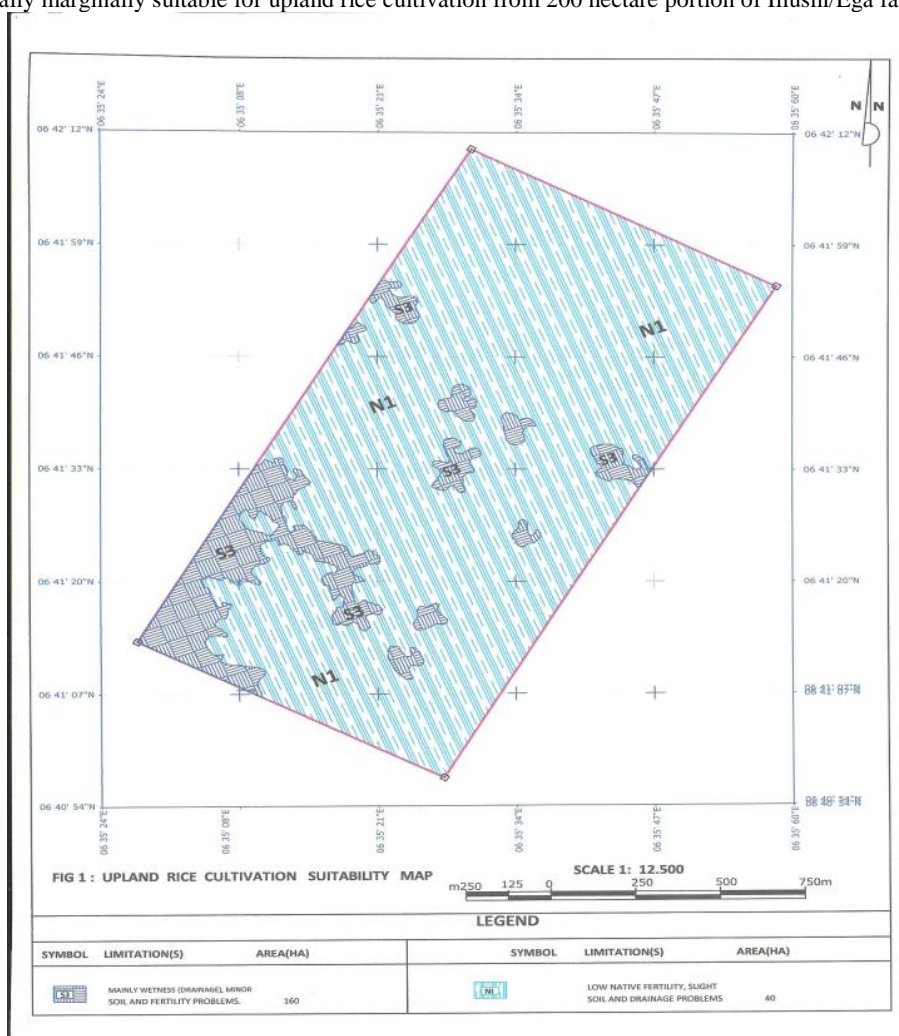




\subsubsection{Irrigated rice cultivation}

The guideline for evaluation of the land for irrigability is in table 3 while table 4 is the outcome of the evaluation efforts and figure 2 is the irrigation suitability map. The results put some 160 hectares $(81 \%$ of the total land area) of the area at marginal suitability with limitations on soil fertility and slightly coarse texture; 11 hectares $(5.5 \%)$ of the area was permanently not suitable because of fertility, coarse textures and therefore excessive drainage limitations that cannot easily and profitably be corrected; and another 29 hectares (14.5\%) found to be currently not suitable with defects bothering on fertility, soils and unfavourable topographic characteristics. While fertility limitations may be easy to correct, soil and topographical defects are usually difficult and often expensive to correct.

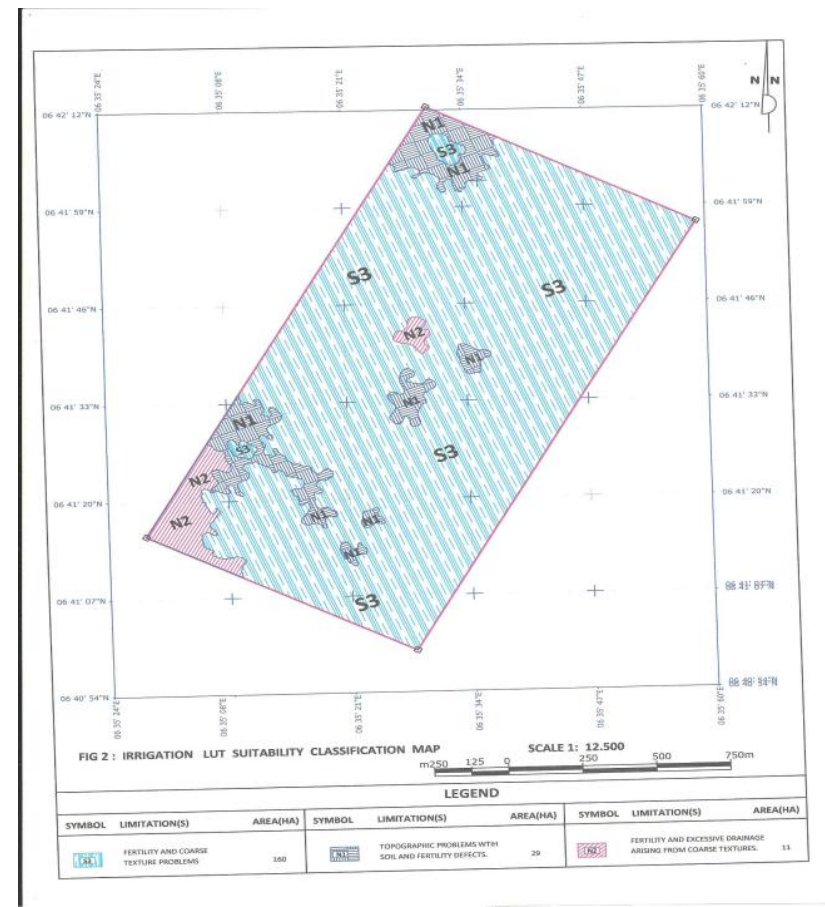

\subsubsection{Rice cultivation using natural floods.}

Table 4 is the guideline for the land evaluation procedure for this LUT and the result is in table 8 and expressed in a suitability map in Figure 3. The results show three levels of suitability. The first is about 55 hectares, found to be moderately suitable for this kind of LUT and with defects in soil fertility, soil, topography and wetness only at moderate levels - i.e. economically profitable to correct. The second is the group of soils that is marginally suitable with limitations in wetness, fertility and topography. This covered some 134 hectares (67\% of total land area). Within this group of soils it is possible that with some drainage measures, the rating of the area occupied by pedon 20/1 (105 ha) can be improved. The third category of soils occupied a small area of 11 hectares $(5.5 \%)$ and has defects that bother on fertility, soil and drainage. The texture is coarse and therefore the drainage is excessive and these are major limitations to the practice of this kind of LUT that cannot easily be corrected and hence the "permanently not suitable" class to which this group was assigned. Thus by current rating, 55 hectares is the real area suitable for this kind of LUT followed by 134 hectares that is marginally suitable. 


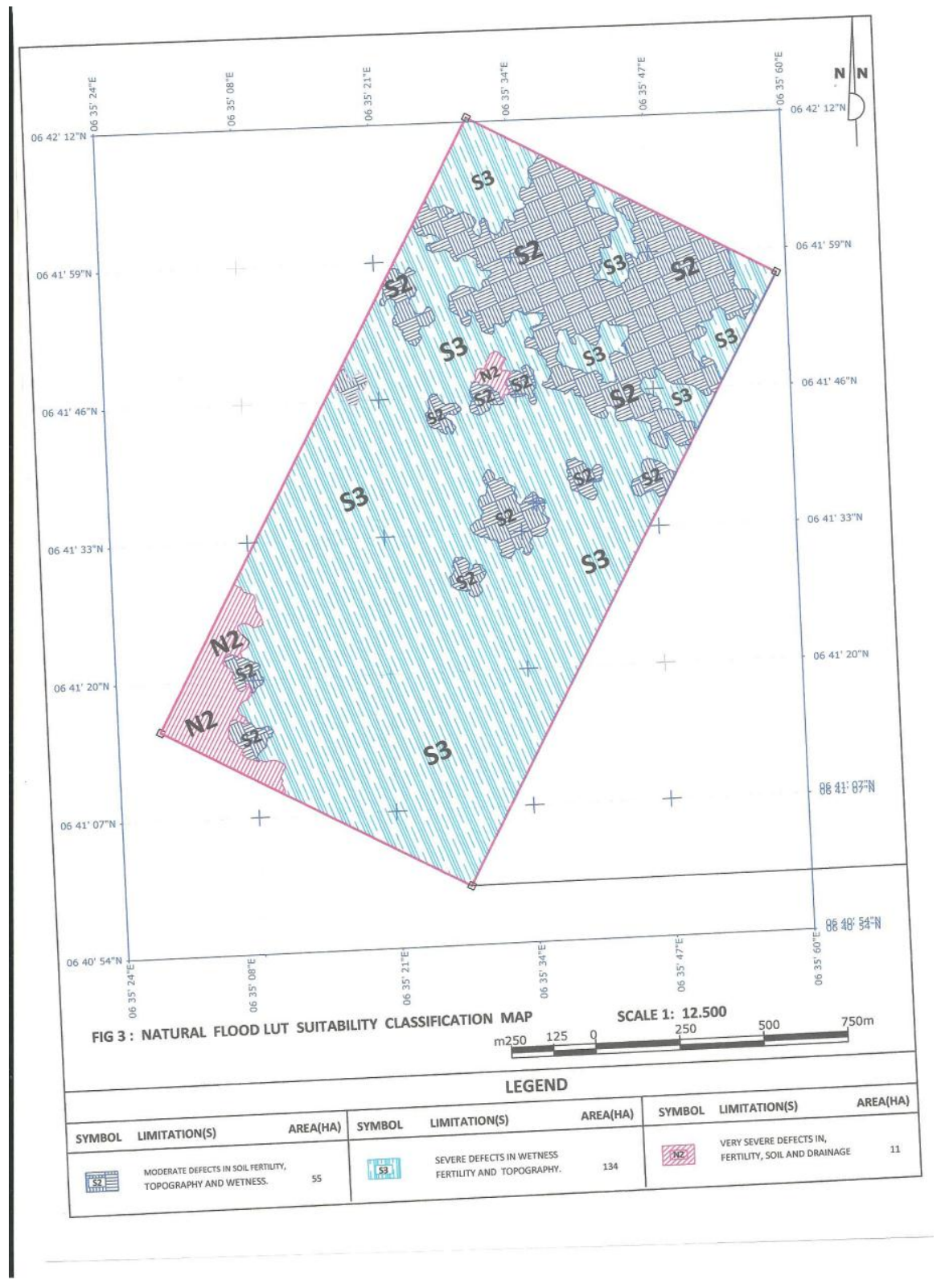

2.5. Comparing the land suitability groupings of the LUTS.

Tables 9,10 and 11 are relevant in these comparisons. Thus it is observed that :

(a) No. of soils in which all three LUTS were equally suitable = Nil;

(b) No. of soils in which two Luts were equally suitable/unsuitable $=5$ (Pedons $3 / 3$ and $4 / 2$ - suitable 55ha; $19 / 11$ - unsuitable; $2 / 9$ and 5/8);

(c) Order of suitability of LUT for the entire land: Natural flood > Irrigated > Upland Rain-fed; and

(d) The best combination of LUT for the usage of that parcel of land would be that of Natural flood followed by Irrigation since both of them use different months of the year and are highly correlated (Table 11).

\section{Conclusions.}

If agricultural production must become attractive to investors, it must become a profitable venture. The first step has to be the ability to guide the investor/farmer on the kind of land utilization that the intended soils can support on a continuous and sustainable basis. This study site cannot sustain the cultivation of upland rainfed rice on a profitable basis. The most appropriate land utilization type, from the outcome of this study is rice production under natural floods in the raining season followed by irrigation practice during the dry season of the year. The 11 hectares occupied by pedon 19/11 can be combined with the other portions for irrigated rice cultivation in the dry season and be left to fallow. This kind of study is useful for all the major crops of Nigeria and other developing countries to provide necessary information for sustainable crop production. 


\section{References.}

[1] FAO (2003). Rice Irrigation in the Near East-Current Situation and Prospects for Improvement. Regional Office of the Near East, Cairo, Egypt. P24.(1)

[2] Genctan, T. (2009). Turkiye' de Celtik uretimi ve sorunlari.I Ulusal Celtik Sempozyumu, Tekirdag - Turkiye.(1)

[3] Longtau, Selbut R. (2003). Nigeria case study report in rice production. Multi-agency partnerships for Technical change in West African Agriculture (MAPS), Jos, Nigeria: Ecosystems development organizations (EDO) for overseas development institute (ODI). (WWW.odi.org.uk/rpeg/maps/nigeria.pdf).( 2)

[4] Tyagi, S. (2003). Agricultural land use planning using remote sensing techniques in part of South GOA (INDIA). Proceedings of the $21^{\text {st }}$ International cartographic conference (ICC). Durban, South Africa, 2003.(2)

[5] FAO (1993). Guidelines for landuse planning. FAO, Rome, Italy. 96p. (2)

[6] Prakash TN. Land Suitability Analysis for Agricultural Crops: A Fuzzy Multicriteria Decision Making Approach. The Netherlands: International Institute for Geo-information Science and Earth observation Enschede; 2003. pp. 6-13. (2)

[7] Dengiz,O.; Sezer,I; Ozdemir, N; Gol, C; Yakupoglu,T; Ozturk, E; Sirat, A; and Sahin, M (2010). Application of GIS model in physical land evaluation suitability for rice cultivation. Anadolu J. Agric. Sci., 2010, 25(S-3):184-191. (2)

[8] Sys, C. (1985). Land evaluation. State university of Ghent, International Training Centre for Postgraduate Soil Scientists, Parts 1,11 , and 111, Ghent.(2)

[9] FAO, (1977b). Guidelines for profile description. Second ed. FAO, Rome.(3)

[10] Bray, R. H. and Kurtz, L. T. (1945). Determination of total, organic and available forms of phosphate in soil. Soil science 59: $225-229 .(3)$

[11] Walkley, A. and Black, I.A. (1934). An examination of the Degtjare method of determining soil organic matter, and a proposed modification of the chromic acid titration method. Soil science 37: $29-38$. (3)

[12] Technicon, AAII. (1971). Technicon Industrial Method, Ammonia in Water and Waste Water. (3)

[13] Tan, K.H. (1996): Soil sampling, preparation and analysis. Marcel Dekker, Inc., New York. 408pp. (3)

[14] Thomas, G.W. (1982). Exchangeable cations. In Page et al (eds). Methods of Soil Analysis, part 2,( agron. Monogr. $2^{\text {nd }}$ ed. ASA and SSSA, Madison, Wisconsin). Pp 159 - 165. (3)

[15] FAO (1984).Guidelines: Land Evaluation for Rainfed Agriculture. FAO Soils Bull. 52: 237. (4) 